\title{
Nonlinear Amplification of Noise in Fibers with Dispersion and Its Impact in Optically Amplified Systems
}

\author{
R. Hui, D. Chowdhury, M. Newhouse, M. O'Sullivan, and M. Poettcker
}

\begin{abstract}
The effect of nonlinear interaction between amplified spontaneous emission noise and dispersive optical fiber material has been investigated analytically. A transfer matrix formulation is used to solve the nonlinear propagation equation. A continuouswave $(\mathrm{CW})$ format of the input optical signal isolates the effect of nonlinear noise amplification from the signal self-phase modulation. The impact of noise amplification in the performance of an intensity-modulation-direct-detection (IM-DD) system is also evaluated.
\end{abstract}

Index Terms-Nonlinear optics, optical fiber communication.

$\mathbf{N}$ ONLINEAR EFFECTS have been reported to be significant in amplified optical fiber transmission systems [1]-[4]. Of particular interest is the Kerr effect, where the refractive index of the fiber medium strongly depends on the intensity of the optical signal.

System performance degradation due to FWM between the signal and amplified spontaneous emission (ASE) has been studied extensively in systems without [5] and with chromatic dispersion [6], [7]. Naka and Saito [7] reported calculations on the change of optical noise spectrum due to Kerr nonlinearity of the fiber. However, in intensity-modulation-direct-detection (IM-DD) optical systems, performance degradation is determined by the relative intensive noise (RIN) induced at the photodetection as has been clarified in [5] and [6]. The aim of this letter is to present a simple analytical method to characterize noise performance in IM-DD nonlinear systems.

Let us begin with the nonlinear Schrodinger Equation [1]

$$
\begin{aligned}
\frac{\partial A(z, t)}{\partial z}= & \frac{-i}{2} \beta^{\prime \prime} \frac{\partial^{2} A(z, t)}{\partial t^{2}}+i \gamma|A(z, t)|^{2} A(z, t) \\
& -\frac{\alpha}{2} A(z, t)
\end{aligned}
$$

where $A$ is the electrical field, $\gamma=\omega n_{2} / c A_{\text {eff }}$ is the nonlinear coefficient of the fiber, $\omega$ is the angular frequency, $n_{2}$ is the refractive index of the fiber, $c$ is the speed of light, $A_{\text {eff }}$ is the effective fiber core area, $\beta^{\prime \prime}$ is the fiber dispersion parameter, and $\alpha$ is the power attenuation coefficient. Highorder dispersions have been ignored here.

The steady-state solution of (1) is $z$-dependent and a simple mean-field approximation is usually not adequate over the

Manuscript received July 1, 1996; revised October 15, 1996.

R. Hui, M. O'Sullivan, and M. Poettcker are with Nortel, Ottawa, Ont., Canada K1Y $4 \mathrm{H} 7$.

D. Chowdhury and M. Newhouse are with Photonics Research, Corning Inc., Corning, NY 14831 USA.

Publisher Item Identifier S 1041-1135(97)01921-6. whole fiber. For an easy solution, the fiber can be divided into short sections and a mean-field approximation can be applied within each section. With the assumption that noise power present at the input of the fiber is much smaller than the signal power, the solution in the $j$ th section can be written as

$$
A_{j}(z, t)=\left[A_{0 j}+\tilde{a}_{j}(z, t)\right] \exp \left(i \gamma_{j}\left|A_{0 j}\right|^{2} z\right)
$$

where $A_{0 j}$ is the steady state solution in this section and $A_{0 j}$ can be chosen to be real such that $A_{0 j}=\left|A_{0 j}\right|$. In the Fourier domain, the evolution of the noise along the fiber can then be calculated simply by matrix multiplication and at the output we have

$$
\left[\begin{array}{c}
a(\omega, L) \\
a^{*}(\omega, L)
\end{array}\right]=\left[\begin{array}{ll}
B_{11} & B_{12} \\
B_{21} & B_{22}
\end{array}\right]\left[\begin{array}{c}
a(\omega, 0) \\
a^{*}(\omega, 0)
\end{array}\right] \exp \left(-\frac{\alpha}{2} L\right)
$$

with

$$
\left[\begin{array}{ll}
B_{11} & B_{12} \\
B_{21} & B_{22}
\end{array}\right]=\prod_{j=1}^{N}\left[\begin{array}{ll}
M_{11}^{(j)} & M_{12}^{(j)} \\
M_{21}^{(j)} & M_{22}^{(j)}
\end{array}\right]
$$

where $L$ is the fiber length, $N$ is the total number of sections, and

$$
\begin{aligned}
M_{11}^{(j)} & =\frac{e^{i k_{j} z}-r_{j}^{2} e^{-i k_{j} z}}{\left|1-r_{j}^{2}\right|} \\
M_{12}^{(j)} & =-M_{21}^{(j)}=\frac{r_{j}\left(e^{-i k_{j} z}-e^{i k_{j} z}\right)}{\left|1-r_{j}^{2}\right|} \\
M_{22}^{(j)} & =\frac{e^{-i k_{j} z}-r_{j}^{2} e^{i k_{j} z}}{\left|1-r_{j}^{2}\right|} \\
r_{j} & =\frac{k_{j}-\beta \omega^{2}-\gamma_{j}\left|A_{0 j}\right|^{2}}{\gamma_{j}\left|A_{0 j}\right|^{2}}=\frac{-\gamma_{j}\left|A_{0 j}\right|^{2}}{k_{j}+\beta \omega^{2}+\gamma_{j}\left|A_{0 j}\right|^{2}}
\end{aligned}
$$

where

$$
k_{j}= \pm \sqrt{\left(\beta \omega^{2}+\gamma_{j}\left|A_{0 j}\right|^{2}\right)^{2}-\left(\gamma_{j}\left|A_{0 j}\right|^{2}\right)^{2}}
$$

is the eigenvalue.

$r_{j}$ is the effective reflectivity for the eigenmode. Therefore, the sign of $\kappa$ should be chosen such that $\left|r_{j}\right|<=1$. $\beta=\beta^{\prime \prime} / 2$ was used in the above equations for simplicity of notation. $\gamma_{j}=\gamma\left[1-\exp \left(-\alpha \Delta z_{j}\right)\right] /\left(\alpha \Delta z_{j}\right)$ is the effective nonlinear coefficient in the $j$ th section.

In an IMDD system, the receiver performance is sensitive only to the amplitude noise of the photo current, in the small signal approximation, the photo current noise is

$$
\delta I(t)=I(t)-I_{0}=\eta A_{0}(L)\left[\tilde{a}^{*}(t, L)+\tilde{a}(t, L)\right]
$$




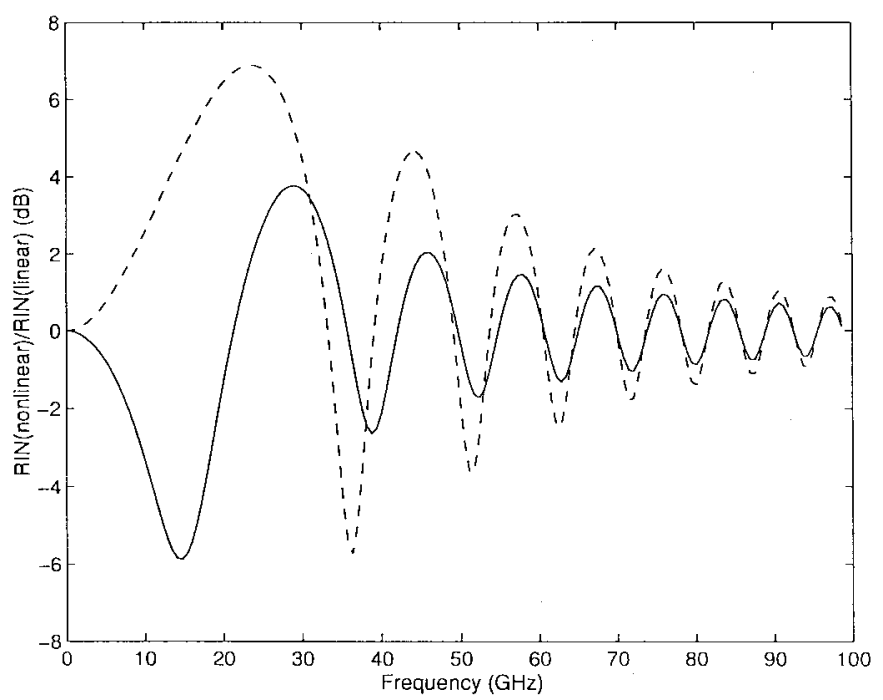

Fig. 1. Normalized relative intensity noise power spectra for normal dispersion (solid line) and anomalous dispersion (dashed line) cases.

where $I_{0}=\eta\left|A_{0}\right|^{2}$ is the photo current generated by the $\mathrm{CW}$ optical signal and $\eta=\eta_{\mathrm{in}} e / h \nu$ is the photodetection responsivity. The power spectrum of the noise photo current is the Fourier transformation of the autocorrelation of the time-domain noise amplitude

$$
\begin{aligned}
\rho_{n}(\omega)= & \eta^{2}\left\{\left|A_{0}^{*} B_{11}+A_{0} B_{21}\right|^{2} S(\omega, 0)\right. \\
& \left.+\left|A_{0}^{*} B_{12}+A_{0} B_{22}\right|^{2} S(-\omega, 0)\right\} e^{-\alpha L}
\end{aligned}
$$

where $S(\omega, 0)$ and $S(-\omega, 0)$ are power spectral of $\widetilde{a}(t, 0)$ and $\tilde{a}^{*}(t, 0)$, respectively.

To simplify the calculation, we have assumed that the input noise spectrum is symmetric around zero frequency (e.g., white noise): $S(\omega, 0)=S(-\omega, 0)$, and it can be proven that $\left|B_{11}+B_{21}\right| 2=\left|B_{12}+B_{22}\right|^{2}$. The increase of the noise power by nonlinear interactions through the fiber is, therefore,

$$
\delta P=\frac{\int_{-\infty}^{\infty}\left|B_{11}+B_{21}\right|^{2} S(\omega, 0) f(\omega) d \omega}{\int_{-\infty}^{\infty} S(\omega, 0) f(\omega) d \omega}
$$

where $f(\omega)$ is the power transfer function of the receiver baseband filter.

The system performance degradation caused by the nonlinear noise amplification can be evaluated through the increase of the standard deviation of noise [9], $d \sigma=\sigma / \sigma_{0}=(\delta P)^{1 / 2}$, where $\sigma$ and $\sigma_{0}$ are standard deviations in the nonlinear and linear cases, respectively. Again, in the Gaussian approximation supposing that the signal extinction ratio is infinite and signal-spontaneous beat noise is the dominant noise source, the system $Q$ degradation caused by the nonlinear noise increase can be expressed as $10 \log (\delta Q)=-5 \log (\delta P)$.

Fig. 1 shows the normalized RIN spectra at the output of an $100-\mathrm{km}$ transmission line with $20-\mathrm{mW}$ input signal optical power. The fiber parameters used are: $\gamma=$ $2.07 \mathrm{~W}^{-1} \mathrm{~km}^{-1}, \alpha=0.22 \mathrm{~dB} / \mathrm{km}, D=+1 \mathrm{ps} / \mathrm{km} / \mathrm{nm}$ (dashed line) and $D=-1 \mathrm{ps} / \mathrm{km} / \mathrm{nm}$ (solid line), where $D=2 \pi c \beta / \lambda^{2}$. It is worth mentioning here that in [8], similar

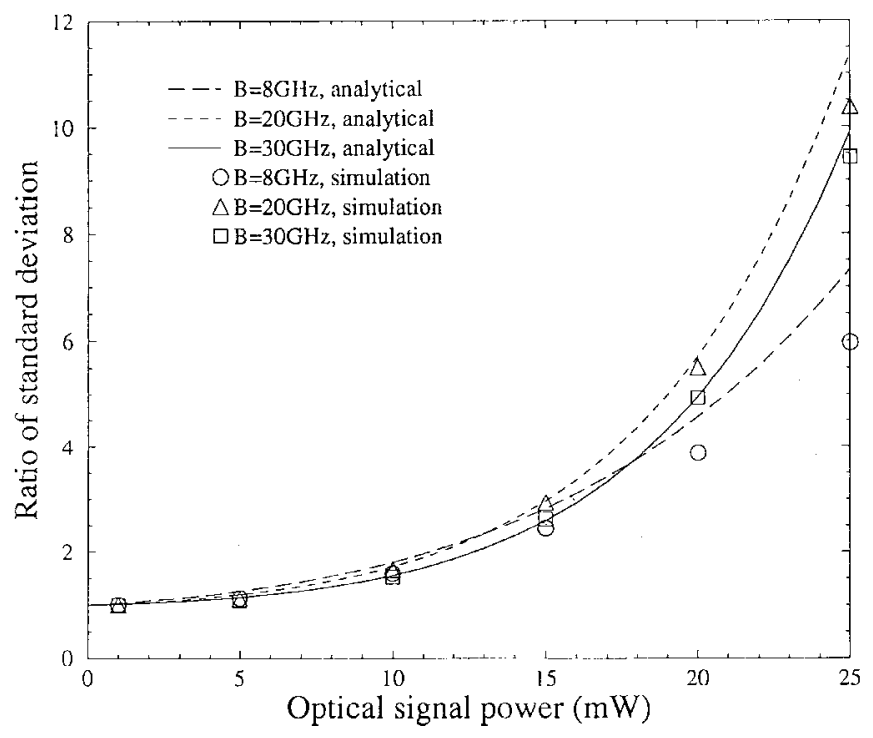

(a)

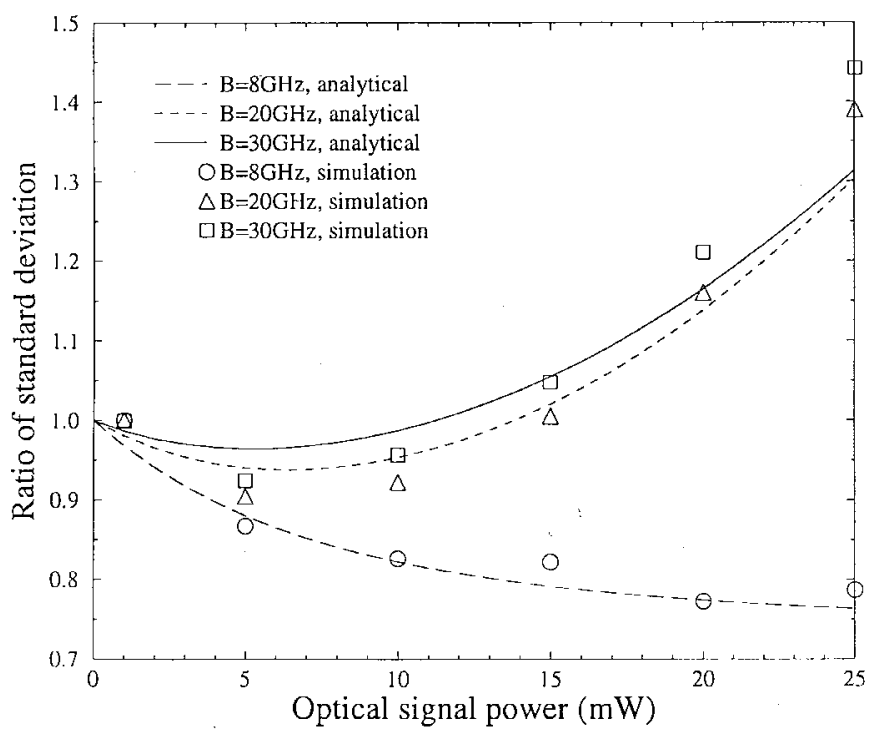

(b)

Fig. 2. Comparison of $\sigma / \sigma_{0}$ between analytical calculation (lines) and computer simulations (open points) for four-span systems with $100 \mathrm{~km}$ per span with fiber dispersion $+2 \mathrm{ps} / \mathrm{km} / \mathrm{nm}$ (a) and $-2 \mathrm{ps} / \mathrm{km} / \mathrm{nm}$ (b). Baseband filter bandwidth are: $8 \mathrm{GHz}$ (long dashed line and circles), $20 \mathrm{GHz}$ (short-dashed line and triangles), and $30 \mathrm{GHz}$ (solid line and squares).

RIN spectra for a lossless fiber have been calculated. However, since the fiber loss was neglected there, the calculated RIN spectra are qualitatively different from those which we have obtained. For example, in the case of normal dispersion, the normalized RIN spectra in [8] are always less than $0 \mathrm{~dB}$ in the mean-field approximation.

In multispan optical amplified systems, since the ASE generated by different erbium-doped fiber amplifiers (EDFA's) are uncorrelated, the performance of the whole system can also be obtained easily. We consider a four-span system as an example, where each span has $100 \mathrm{~km}$ of fiber. In order to make a quantitative evaluation of the system performance, Fig. 2(a) and 2(b) shows the ratio of noise standard deviation between nonlinear and linear cases for $D=+2 \mathrm{ps} / \mathrm{km} / \mathrm{nm}$ 


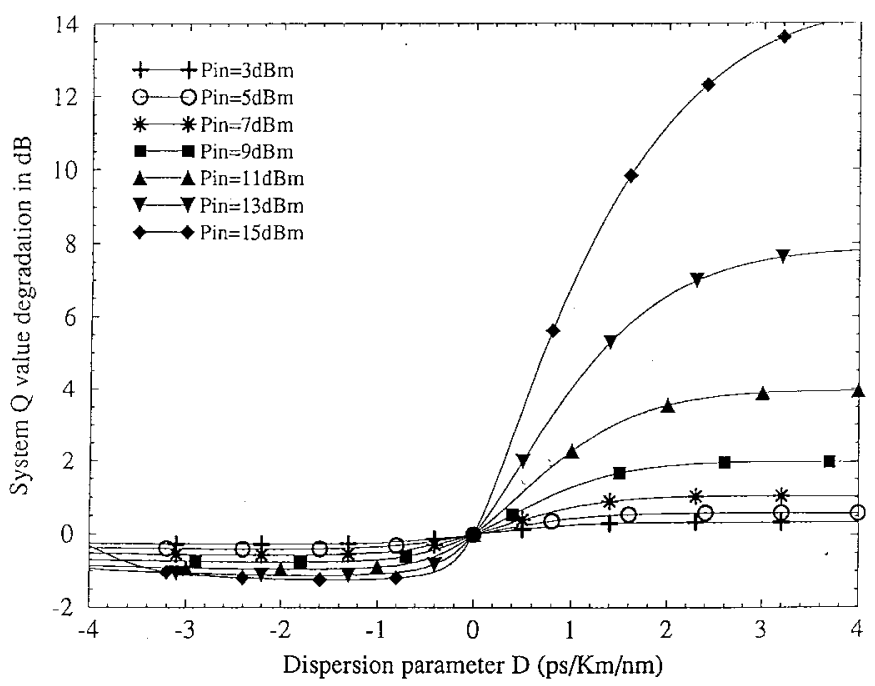

Fig. 3. Nonlinear degradation of system $Q$ versus fiber dispersion for different signal power levels. Electrical bandwidth $8 \mathrm{GHz}$, four-span system with $100 \mathrm{~km}$ per span.

and $D=-2 \mathrm{ps} / \mathrm{km} / \mathrm{nm}$, respectively. Raised-cosine filters are used in the calculation with three different bandwidths, among which the $8-\mathrm{GHz}$ bandwidth corresponds to a $10-\mathrm{Gb} / \mathrm{s}$ (OC-192) system, and $30 \mathrm{GHz}$ corresponds to a 40-Gb/s (OC768) system. It is interesting to note that in the anomalous dispersion regime, system sensitivity always becomes worse with increasing signal power. On the other hand, in the normal dispersion regime, system noise performance can be improved by the nonlinear process for an OC-192 system. However, for systems with higher bit rate, sensitivity degradations are also possible in the normal dispersion regime with high input signal power. This degradation is believed to be caused by the subsidiary peaks as shown in Fig. 1 (solid line). In order to verify the validity of the transfer matrix method, computer simulations were also conducted which solve the nonlinear pulse propagation equations with the use of the split-step Fourier method [1]. Computer simulation results given in Fig. 2 show a reasonable agreement with the transfer matrix results. Small deviations are believed to originate from the random behavior of the noise generator in the simulation.

Fig. 3 shows the $Q$ degradation of an $10-\mathrm{Gb} / \mathrm{s}$ system. The results indicate that signal optical power should be kept less than $7 \mathrm{~dB} \cdot \mathrm{m}$ to maintain $Q$ degradation of less than $1 \mathrm{~dB}$ in this case. It is easy to see from this plot that at zero dispersion, system performance degradation is always zero irrespective of optical signal power. This agrees with results of early studies [5], [6] made under the assumption of unlimited system optical bandwidth. However, in ultra long fiber optical systems operating at zero dispersion, the nonlinearly-broadened optical spectrum spreads well beyond system effective optical bandwidth, creating large penalties [5]. The linear approximation fails in that extreme situation. Performance degradation caused by nonlinear noise amplification versus the number of optical amplified spans for $10-\mathrm{Gb} / \mathrm{s}$ systems were also calculated and reported in Fig. 4.

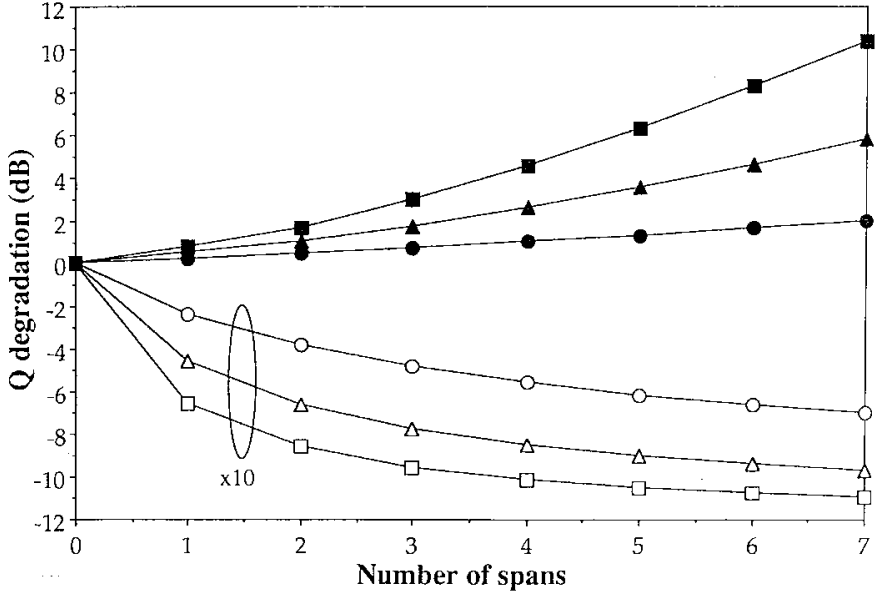

Fig. 4. Nonlinear degradation of system $Q$ versus the number of fiber spans for different signal power levels. Electrical bandwidth: $8 \mathrm{GHz}, 100 \mathrm{~km}$ per span. Open circles: $D=-2 \mathrm{ps} / \mathrm{km} / \mathrm{nm}$ and $P_{\text {in }}=5 \mathrm{~mW}$. Open triangles: $D=-2 \mathrm{ps} / \mathrm{km} / \mathrm{nm}$ and $P_{\text {in }}=10 \mathrm{~mW}$. Open squares: $D=-2 \mathrm{ps} / \mathrm{km} / \mathrm{nm}$ and $P_{\text {in }}=15 \mathrm{~mW}$. Solid circles: $D=2 \mathrm{ps} / \mathrm{km} / \mathrm{nm}$ and $P_{\text {in }}=5 \mathrm{~mW}$. Solid triangles: $D=2 \mathrm{ps} / \mathrm{km} / \mathrm{nm}$ and $P_{\text {in }}=10 \mathrm{~mW}$. Solid squares: $D=$ $2 \mathrm{ps} / \mathrm{km} / \mathrm{nm}$ and $P_{\text {in }}=15 \mathrm{~mW}$.

In conclusion, a transfer matrix model has been developed to analyze the impact of nonlinearity on the ASE noise for multispan fiber systems. The results from this model compare well with simulation results which are obtained by solving the nonlinear propagation equation numerically. Even though it is well known that Kerr nonlinearity can improve system performance through signal SPM [3], [10], we demonstrated that for systems operating at normal dispersion, nonlinearity reduces the deleterious impact of ASE noise when compared with linear propagation. This improvement is achieved over a range of signal power level and receiver bandwidth. However, in the anomalous dispersion regime, nonlinearity always degrades the system performance when compared with linear propagation.

\section{REFERENCES}

[1] G. P. Agrawal, Nonlinear Fiber Optics. New York: Academic, 1989.

[2] A. Chraplyvy, "Limitations on lightwave communications imposed by optical-fiber nonlinearities," IEEE J. Lightwave Technol., vol. 8, pp 1548-1557, 1990.

[3] N. Suzuki and T. Ozeki, "Simultaneous compensation of laser chirp, Kerr effect, and dispersion in 10-Gb/s long-haul transmission systems," IEEE J. Lightwave Technol., vol. 11, pp 1486-1494, 1993.

[4] D. Marcuse, "Single-channel operation in very long nonlinear fibers with optical amplifiers at zero dispersion." IEEE J. Lightwave Technol., vol. 9, pp 356-361, 1991.

[5] A. Mecozzi, "Error probability of amplified IMDD systems at zero dispersion." Electron. Lett., vol. 24, pp. 2136-2137, 1993.

[6] D. Marcuse, "Noise properties of four-wave mixing of signal and noise," Electron. Lett., vol. 30, pp1175-1177, 1994.

[7] A. Naka and S. Saito, "Noise evolution in in-line amplifier systems due to the nonlinear refractive index of optical fibers," in Proc. OFC'94, pp. 287-288.

[8] M. Yu, G. P. Agrawal, and C. J. McKinstrie, "Pump-wave effects on the propagation of noisy signals in nonlinear dispersive media," J. Opt. Soc. Am. B, vol. 11, pp. 1126-1132, 1995.

[9] D. Marcuse, "Derivation of analytical expressions for the bit-error probability in lightwave systems with optical amplifiers," IEEE $J$. Lightwave Technol., vol. 8, pp 1816-1823, 1990.

[10] M. Schiess, "Chirp and dispersion compensation in nonlinear fibers for high bit-rate IM/DD systems," in Proc. ECOC'93, pp. 481-484. 